Case Report

\title{
Unusual Presentation of Fungal Keratitis- Anterior Chamber Granuloma with Necrotising Scleritis : A Case Report
}

\section{Soumya Haridasan ${ }^{1} \&$ Vijay Pai $^{2}$}

${ }^{1}$ Postgraduate, ${ }^{2}$ Professor and HOD, Department of Ophthalmology, K. S. Hegde Medical Academy, Nitte University, M angalore, Karnataka, India.

*Corresponding Author : Soumya Haridasan, Post graduate, Department of Ophthalmology, K. S. Hegde Medical Academy, Nitte University, M angalore- 575018, Karnataka, India.

Mobile : +9199164 82793,+919745697244 E-mail : drsoumya06@yahoo.com

Received

: 24-12-2015

Review Completed : 18-04-2016

Accepted

: 19-04-2016

Keywords : Fungal keratitis, anterior chamber granuloma, intracameral voriconazole, intrastromal voriconazole

\begin{tabular}{|c|}
\hline Access this article online \\
\hline Quick Response Code \\
\hline
\end{tabular}

\begin{abstract}
:
This is a rare case of fungal keratitis presenting as anterior chamber granuloma with necrotising scleritis where a 56 year old woman presented with pain, redness, watering, discharge and blurring of vision in right eye for 6 months and on examination was found to have granulomatous exudate with hypopyon in anterior chamber and staphyloma. On treatment with Intracameral and intrastromal voriconazole the symptoms subsided within a week. This treatment modality proved to be helpful in preventing the progression of such a blinding disease and improving the vision.
\end{abstract}

\section{Introduction}

Fungal keratitis is a leading cause of monocular blindness worldwide with a reported incidence varying from 6 to $50 \%$ and a tendency to significantly increase in tropical climates ${ }^{1}$. Predisposing factors are trauma with organic matter, long-term use of broad-spectrum antibiotics and topical steroid, corneal surface disorders, refractive surgery and specific contact lens disinfectant solutions ${ }^{2}$. Several studies have indicated variable responses to a number of conventional topical and systemic antifungal agents $^{3,}{ }^{4}$. Despite aggressive therapy, the keratitis, especially of deeper corneal layers occasionally progress to endophthalmitis or panophthalmitis regardless of underlying conditions because of poor corneal penetration and limited spectrum of conventional antifungal agents $s^{5,6}$. Recently there has been several studies evaluating the use of intracameral voriconazole, a newer generation triazole, to manage a case of infectious keratitis with successful outcomes ${ }^{6,7,8}$. Here we report one such rare case of fungal keratitis treated with intracameral and intrastromal voriconazole.

\section{Case Report}

A 56 year old female presented with pain, redness, watering, discharge and blurring of vision in right eye since 6 months following trauma to the eye with a wooden stick. Slit-lamp examination revealed eye lid edema, ciliary and conjunctival congestion, temporal scleral thinning with staphyloma, an yellowish-white mid to deep stromal infiltrate at the temporal cornea with intact epithelium, a granulomatous exudate temporally underlying which a large exudates was seen in the anterior chamber with a hypopyon of $\sim 1 \mathrm{~mm}$. (Fig $1 \mathrm{a}$ and $1 \mathrm{~b}$ ). 


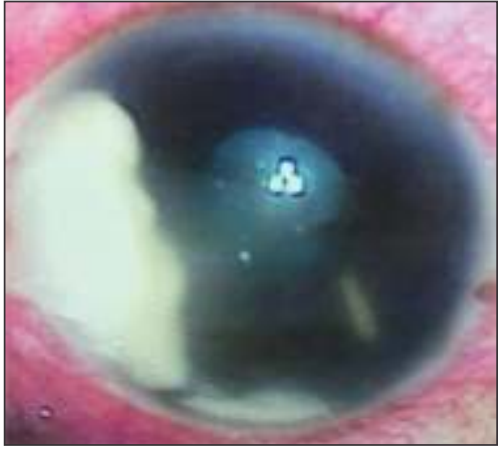

Figure 1a : A temporal granulomatous exudate with $1 \mathrm{~mm}$ hypopyon in anterior chamber

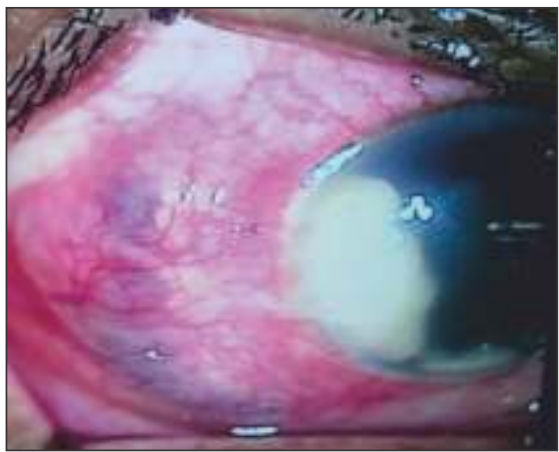

Figure 1b: Temporal staphyloma

The exudates were removed through a paracentesis and with (Fig 2) and sent for microbiological analysis. Intracameral and intrastromal Voriconazole $(50 \mu \mathrm{g}$ in $0.1 \mathrm{ml}$ ) was injected and surgery completed. (Fig 3).

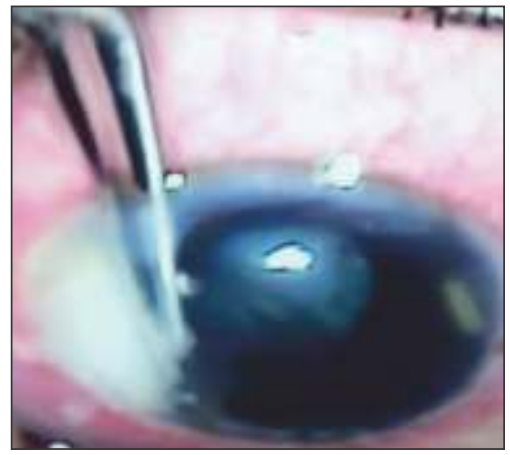

Figure 2 : Removal of exudates via paracentesis created at 10'o clock.

\section{Discussion}

Voriconazole is a new second generation triazole derived from fluconazole that has demonstrated effectiveness against fungal keratitis caused by a broad range of fungal pathogens $s^{9,10}$. It primarily inhibits the cytochrome P450 14alpha demethylase and 24-methylene dihydrolanasterol demethylation in certain yeasts and filamentous fungi and has a molecular mass of 349.32 Da that allows good corneal penetration ${ }^{11}$ and therefore better ocular bioavailability ${ }^{1}$.

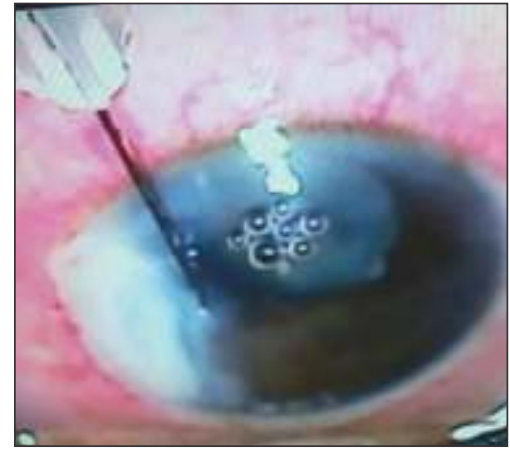

Figure 3: Intracameral and intrastromal injection of voriconazole

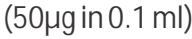

Microbiological evaluation showed multiple septate fungal filaments on gram stain and Potassium Hydroxide $(\mathrm{KOH})$ stain and fungal colonies in Sabouraud Dextrose Agar (SDA) and blood agar. Post-operatively patient was managed with hourly Voriconazole $1 \%$ eye drops, hourly Natamet $5 \%$ eye drops, M oxicip $0.5 \%$ eye drops 6 times/day, Homide 1\% eye drops 3times/day and systemic antibiotic and antifungal medications such as I.V.Tazar (piperacillin + tazobactam) 2.25 mg t.i.d and oral Ketoconazole $200 \mathrm{mg} \mathrm{b.d}$. On follow up, patient's symptoms subsided and healed with improvement in vision (Fig 4).
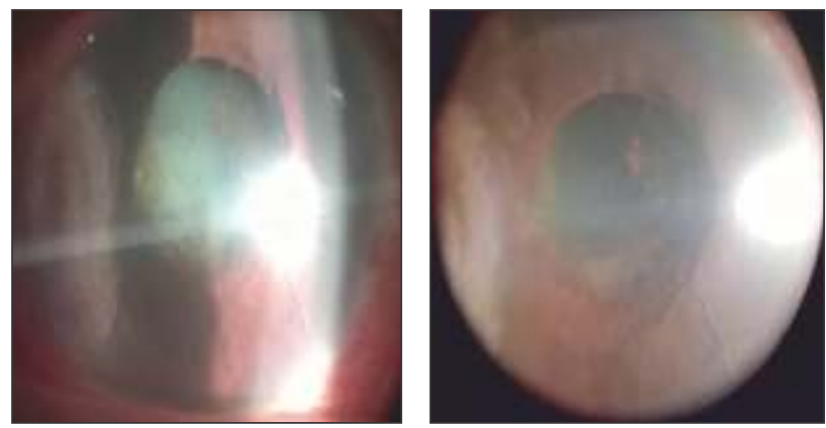

Figure 4 : 1week postoperative : Healed fungal ulcer following intracameral and intrastromal voriconazole injection

With topical administration, voriconazole has shown excellent penetration through the cornea into the aqueous humor, without compromising intraocular safety ${ }^{10}$ Although voriconazole possesses excellent bioavailability and the therapeutic aqueous level can be achieved after topical administration, ${ }^{9,}$, there might be some difficulties in maintaining effective drug concentrations at the site of lesions ${ }^{13}$. Therefore, targeted drug delivery in the form of intrastromal and intracameral injection may have a role in treating keratomycosis ${ }^{14}$.

Numerous studies have investigated the effect of targeted drug deliveries using intrastromal or intracameral injections of this potent drug and found that these less invasive surgical modalities were safe, very effective, and 
well tolerated, and proved to be significant in improving visual prognosis si,15 $^{12}$

The experimental use of intracameral voriconazole in humans showed no toxic effects when the aqueous concentration was $(10 \mu \mathrm{g} / \mathrm{mL}-1.5 \mathrm{mg} / \mathrm{mL}){ }^{16}$ Above 1.5 $\mathrm{mg} / \mathrm{mL}$ there was a dose-dependent reduction in corneal endothelial cells, trabecular meshwork cells, and retinal pigment epithelial cells ${ }^{16}$.

Our case had granulomatous exudates with hypopyon similar to one of the cases in the study by Solaiman KA et al $(2015)^{7}$. having central thick plaque with hypopyon and Mittal V et al (2012) ${ }^{6}$. Having endoexudates, which used

\section{References}

1. Haddad SR, El-Mollayess GM. Combination of Intracameral and Intrastromal Voriconazole in the Treatment of Recalcitrant Acremonium Fungal Keratitis. Middle East African Journal of Ophthalmology, 2012; 19(2):265-8.

2. Srinivasan M. Fungal keratitis. Curr Opin Ophthalmol. 2004; 15:321-7.

3. Prajna NV, Krishnan T, Mascarenhas J, et al. The mycotic ulcer treatment trial: a randomized trial comparing natamycin vs voriconazole. JAM A Ophthalmol. 2013; 131:422-429.

4. Mahdy RA, WM Nada, MM Wageh. Topical amphotericin B and subconjunctival injection of fluconazole (combination therapy) versus topical amphotericin B (monotherapy) in treatment of keratomycosis. J Ocul Pharmacol Ther. 2010 jun; 26(3):281-5.

5. Lekhanont K, Nonpassopon M, Nimvorapun N, Santanirand P. Treatment With Intrastromal and Intracameral Voriconazole in 2 Eyes With Lasiodiplodia theobromae Keratitis. Medicine. 2015; 94(6):1-6.

6. M ittal V, Mittal R. Intracameral and Topical Voriconazole for Fungal Corneal Endoexudates. Cornea 2012; 31(4):366-370.

7. Solaiman KA, Alkawas AA, Ibrahim BM, Mahdy MM, Shalaby MA (2015) Topical Voriconazole Drops With and Without Intrastromal Voriconazole Injection for Treatment of Deep or Resistant Fungal Keratitis. J Clin Exp Ophthalmol 6: 469.

8. Shen YC, Wang CY, Tsai HY, Lee HN. Intracameral voriconazole injection in the treatment of fungal endophthalmitis resulting from keratitis. American journal of ophthalmology. 2010 jun; 149(6):916-21. intrastromal and intracameral voriconazole respectively for the treatment of fungal keratitis.

In our case, intrastromal and intracameral injections of $50 \mu \mathrm{g} / 0.1 \mathrm{~mL}$ voriconazole was administered following which the outcome was successful with healing of the ulcer and good visual acuity. This was in concordance with the study by Lekhamont et al $(2015)^{5}$ and Haddad et al (2012) ${ }^{1}$.

\section{Conclusion}

Fungal keratitis can masquerade as anterior chamber granuloma which can be effectively managed by using intracameral and intrastromal voriconazole.

9. Hariprasad SM , M ieler WF, Lin TK, et al. Voriconazole in the treatment of fungal eye infections: a review of current literature. $\mathrm{Br} J$ Ophthalmol. 2008; 92:871-878.

10. Al-Badriyeh D, Neoh CF, Stewart K, et al. Clinical utility of voriconazole eye drops in ophthalmic fungal keratitis. Clin Ophthalmol.2010; 4:391-405.

11. Chandrasekar PH, Manavathu E. Voriconazole: A second generation triazole. Drugs Today (Barc) 2001; 37:135-48

12. Sharma N, Chacko J, Velpandian T, et al. Comparative evaluation of topical versus intrastromal voriconazole as an adjunct to natamycin in recalcitrant fungal keratitis. Ophthalmology. 2013; 120:677-681.

13. Prakash G, Sharma N, Goel M, et al. Evaluation of intrastromal injection of voriconazole as a therapeutic adjunctive for the management of deep recalcitrant fungal keratitis. Am J Ophthalmol. 2008; 146:56-59.

14. Sharma N, Agarwal $P$, Sinha R, et al. Evaluation of intrastromal voriconazole injection in recalcitrant deep fungal keratitis: case series. Brj Ophthalmol. 2011; 95:1735-1737.

15. Kalaiselvi G, Narayana S, Krishnan T, et al. Intrastromal voriconazole for deep recalcitrant fungal keratitis: a case series. $\mathrm{Br} J$ Ophthalmol. 2015; 99:195-198.

16. Kernt M, Kampik A. Intracameral voriconazole: In vitro safety for human ocular cells. Toxicology 2009; 258:84-93. 